\title{
EL FONEMA /S/ EN EL HABLA DE LA CRUZ, SINALOA
}

Tan pronto como se tiene contacto con el habla de la costa noroeste $^{1}$ de México, sorprende notablemente un fenómeno fonético: las diversas realizaciones de $s, y$, más específicamente, su aspiración.

Anteriormente, en mis experiencias de campo por otros lugares de la República, concretamente por los estados de Veracruz y Tabasco (costa atlántica) y por los estados de Guerrero y Oaxaca (costa del Pacífico), había notado diferencias en la aspiración de $/ \mathrm{s} /{ }^{2}$ de una costa a otra. Sin embargo, esta realización, la de Sinaloa, era diferente a todo lo oído en otras regiones, por lo que me propuse hacer un estudio bastante estricto, en el sentido estadístico; los resultados son los que presento.

He elegido La Cruz, Sinaloa ${ }^{3}$, porque es la localidad donde con

1 A principios de 1974, en el Seminario de Dialectología de El Colegio de México, dentro del proyecto de "Delimitación de las Zonas Dialectales", se programó el trabajo de campo de una parte de la costa noroccidental de la República Mexicana. El trabajo se realizó del 10 al 26 de marzo de ese mismo año, y comprendió las localidades de Mazatlán, La Cruz, Culiacán, Guamúchil y Los Mochis, en el estado de Sinaloa, y Navojoa, Alamos y Ciudad Obregón, en el sur del estado de Sonora. Con el profesor G. Cantero llevé a cabo las encuestas.

2 Tras el análisis fonético de las grabaciones magnetofónicas de estas regiones, aumentó mi sospecha acerca de que en México existian por lo menos dos tipos de aspiración condicionados por la distribución complementaria. $\mathrm{Pa}$ rece ser que en el Golfo de México la aspiración de $/ s /$ es más frecuente ante consonante sorda, mientras que en el Pacífico parece más frecuente ante sonora (vocal o consonante). No he podido hacer un estudio a fondo de varias localidades de ambas costas, por falta de tiempo, pero este condicionamiento resulta bastante interesante.

3 La Cruz es cabecera de municipio; en 1970 contaba con 4218 habitantes, y en la actualidad tiene aproximadamente 5000 . Tiene dos escuelas primarias y solamente una escuela secundaria. Como en todas las encuestas válidas para el proyecto de Zonas Dialectales, aplicamos tres cuestionarios (véase el Cuestionario para la delimitación de las zonas dialectales de México, México, 1970; más noticias sobre el proyecto se pueden encontrar en la $N R F H, 19$, 
más claridad percibí el fenómeno, sobre todo en las grabaciones magnetofónicas, y donde los habitantes, al parecer, tienen más conciencia de él. Recuerdo que la máxima autoridad del poblado, el presidente municipal, profesor normalista de enseñanza primaria, me señaló claramente que en La Cruz no decían [masatlán] sino [mahatlán] y un grupo de campesinos que lo acompañaba corroboró lo dicho.

\section{GRABACIONES}

1. Ma. Luisa Bastidas, nacida en el municipio (Agua Nueva, Sin.), al igual que sus padres y esposo. 46 años. Analfabeta (segundo año de primaria). No ha viajado. Ocupación: hogar.

2. José Salazar Imperial, nacido en La Cruz, su padre en un pueblo cercano: El Tepuyo, y su madre en Elota, a $20 \mathrm{~km}$. de distancia. 24 años. Semianalfabeto (5 $5^{\circ}$ año de primaria). Viajes breves por la región. Campesino de ocupación. Estado civil: soltero.

3. Carmen Rodríguez García, nacida en el municipio (Agua Nueva, Sin.) al igual que sus padres. 30 años. Nivel sociocultural medio (4año de primaria). Viajes por la región y una residencia de tres meses en Los Ángeles, California, E.U.A. Ocupación: hogar. Estado civil: soltera.

4. Leoncio Aguilar Barraza, nacido en Elota (antigua cabecera municipal), al igual que sus padres. 81 años. Nivel sociocultural medio ( $6^{\circ}$ año de primaria). Ha viajado por la región y residió varios años, en su juventud, en los E.U.A. Ex empleado federal, en la actualidad es pensionado.

\section{Cuestionarios}

1. César Delgado Toscano, nacido en La Cruz; sus padres, nacidos en Cocoyol, Dgo: y su esposa también natural de La Cruz. 32 años. Semianalfabeto ( $3^{\circ}$ de primaria). Ha ido de paseo a E.U.A. Trabaja como policía.

2. Jesús Ozuna Bracamontes, nacido en Elota, al igual que sus padres; su esposa, nacida en El Salto, Sin. 54 años. Semianalfabeto (4 año de primaria). Residió durante 4 meses en E.U.A. Ocupación: ferrocarrilero.

3. Rosa Rodríguez Vda. de Franco, nacida en el municipio (Agua Nueva, Sin.), al igual que su madre y su esposo; padre oriundo de Guadalajara, Jal. 79 años. Nivel sociocultural medio $\left(4^{\circ}\right.$ año 
de primaria). Ha viajado por la República y E.U.A. Su esposo era empleado público y ella se ha dedicado al hogar.

\section{Metodología:}

Fundamento mis observaciones sobre todo en el análisis de las grabaciones magnetofónicas; he tomado en cuenta los cuestionarios solamente para una última comparación a manera de datos complementarios.

He desarrollado el estudio de estas grabaciones de la siguiente manera: de acuerdo con el análisis tradicional en el seminario de "Zonas Dialectales" consideré las siguientes posiciones posibles de $/ \mathrm{s} /$ :

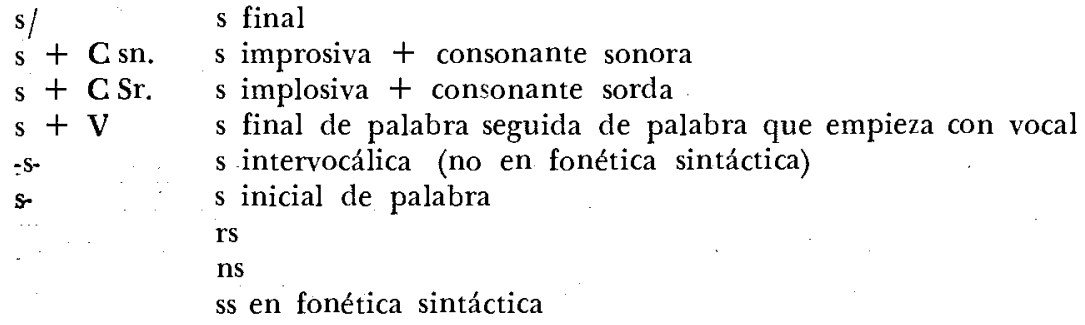

De cada grabación anoté todas las $s$ que aparecieron en quince minutos de la encuesta, busqué la distribución que presentaban y las coloqué en su apartado específico. De esta forma obtenía en cada posición porcentajes bastante cerrados de la variación alofónica. Esto no quiere decir que los porcentajes sean un reflejo matemáticamente perfecto en relación con la totalidad del habla de cada informante, ya que existen las variantes contextuales. La ocasión, el momento, el interlocutor, etc., pueden ser factores para un cambio de la realización de cualquier fonema.

A continuación presento un cuadro estadístico que muestra las veces que cada informante realiza un alófono cualquiera en una distribución determinada, los subtotales y totales de apariciones alofónicas.

La primera columna, "Posición", indica la distribución que ocu pa el fonema en la palabra o en el discurso en casos de fonética sintáctica: final absoluta, implosiva ante sonora, etc. La segunda reúne los alófonos registrados. De la tercera a la sexta columna se señala la frecuencia, el tanto por ciento con que el informante realizó un alófono determinado, y, finalmente, el séptimo apartado indica el promedio porcentual de realización de cada alófono.

Si se considera este sondeo matemático que he realizado como representativo del habla de la localidad, puedo decir que el fone- 
$\mathrm{ma} / \mathrm{s}$ / en La Cruz, en general, es sumamente polimórfico: se produce predorso alveolar, dentalizado, aspirado, etc.

Globalmente puedo decir que cuando la /s/ no se realiza como una aspiración laríngea - cosa que, entre paréntesis, no es muy frecuente en relación con otros lugares $(26.12 \%)-$, se puede decir que oscila entre tres tipos de /s/ bien documentados tanto en México como en toda América y aun en el español en general; la preferencia por un tipo determinado se debe sobre todo a la tensión: una tensión débil tiende a un tipo, si aumenta resulta otra $s$ diferente. Se registra el tipo coronal dentoalveolar plana ( $)$, o sea el mismo tipo que se puede documentar en distintos países de América y en los estados norteños de México ${ }^{4}$; también encontramos la predorsoalveolar convexa con apoyo de la punta de la lengua en los incisivos inferiores (s), tan frecuente en el Valle de México; otra variante es la dental ligeramente convexa más aguda que la plana por ser el contacto de la lengua con los dientes, más con el ápice que con la corona $(\$)$.

Ahora bien, analizando detalladamente y por partes los datos, se puede llegar a distintas conclusiones provisionales sujetas a indagaciones más amplias. En forma de hipótesis puedo señalar las siguientes características:

1) La aspiración es un fenómeno propio del habla masculina de los jóvenes. La aspiración de $s$ suele ser más frecuente entre los hombres que entre las mujeres, y más entre los jóvenes que entre los viejos: el informante II, que es un hombre joven de veinticuatro años, es quien más aspira, aún más que el informante IV, anciano de 81 años. Ambos aspiran más que las mujeres (Infs. I y III), aunque la mujer joven (Inf. III, 30 años) aspira más que la mujer madura (Inf. I, 46 años). Creo que el nivel sociocultural no influye en este fenómeno, pues el Inf. II, que es el que más aspira, es semianalfabeto, mientras que el Inf. I, que es quien menos aspira, pertenece al nivel analfabeto. Más claro, el hombre joven (Inf. II) es semianalafabeto y aspira más que el hombre viejo (Inf. IV) que tiene un nivel medio más bien alto. Esto nos llevaría a pensar que las clases bajas aspiran más, pero la mujer joven (Inf. III), de nivel medio, aspira más que la mujer vieja (Inf. II), de nivel analfabeto,

4 Es "El mismo tipo que se encuentra en Sto. Domingo, en Colombia y en todas las Antillas, en las costas y en los estados norteños de México, en Nuevo México y en gran parte de Andalucía" (J. H. Matluck, "Fonemas finales en el consonantismo puertorriqueño", $N R F H, 15,1961,332-342$; véase también Ch. C. Marden, "La fonología del español en la ciudad de México", $B D H, 4$, 1938, \$ 35, nota 3. P. Henríquez UREÑA, "Mutaciones articulatorias en el habla popular", ibid., $\$ 4$ y, sobre todo, J. MATLuck, La pronunciación en el español del Valle de México, México, 1951, \$117). 
pero menos que el Inf. IV que es de nivel sociocultural superior. Si el estrato condicionara la aspiración, los informantes I y II serían quienes más aspiraran, a continuación el Inf. III y el Inf. IV sería el que menos aspirara; o sea, el orden por el grado de aspiración sería: I, II, III, IV, cuando en realidad es: II, IV, III, İ.

2) La aspiración de $s$ final absoluta es inferior a la aspiración de $s$ en otras posiciones implosivas. Teniendo en cuenta que en el habla de la localidad se registra aspiración de $s$, podría esperarse que la $s$ en posición final absoluta se aspirara abundatemente; sin embargo, como puede observarse, no sucede así; la /s/ final absoluta se realiza sin aspiración $(80.69 \%)$ : es predorsoalveolar $(61.23 \%)$, o dental $(s-8.8 \%)$, o plana $(\varsigma-7.92 \%)$, o bien nasalizada $\left(\mathrm{s}^{\mathrm{n}}\right.$ $-2.64 \%$ ). En un $19.38 \%$ (44 de 227 casos) se puede considerar aspirada ${ }^{5}$. Aún es más notorio que el Inf. I y el Inf. III casi no aspiran en esta posición, sólo 3 de 75 posibles y 2 de 47 para un promedio escaso del $4.09 \%$. Aún el Inf. IV apenas alcanza un $15.38 \%$ de realizaciones aspiradas. Esto parece caracterizar al dialecto de La Cruz, pues hasta donde he podido investigar, en todas las regiones donde hay aspiración, la posición más favorecida es la final, que puede darse como aspiración o como pérdida absoluta: "En posición final ante pausa -dice T. Navarro-, la $s$ puertorriqueña desaparece normalmente en el habla popular... La supresión de la $s$ final, extendida por toda la isla, se oye hasta en el habla familiar de las personas instruidas" ${ }^{6}$.

3) La aspiración de $s$ es favorecida por el contacto con sonora. En posición implosiva y ante consonante sonora es bastante notoria la aspiración, llega a alcanzar hasta el $81.25 \%$ y el resto se reparte entre los tres tipos de $s$ señalados anteriormente. Si se observa la posición ante consonante sorda advertimos que el grado de aspiración baja notablemente, por lo que se puede sospechar que, siendo la sonorización al igual que la aspiración una forma de debilitación ${ }^{7}$, en este dialecto aquélla favorece a ésta; es decir, la aspiración en esta región parece ser favorecida más que por la "distensión de la sílaba”, por el contacto con fonemas sonoros. Esto parece explicar

5 Como aspirados he considerado todos aquellos alófonos que presentan siquiera un mínimo grado de aspiración.

6 El español en Puerto Rico, 2a ed., Río Piedras, 1966, p. 73; véase también J. MATLuck, op. cit., \$126, nota; L. FlóREZ, La pronunciación del espanol en Bogotá, Bogotá, 1951, § 88.1; A. M. Espinosa, "Estudios sobre el español de Nuevo México", $B D H, 4$ (1938), p. 19; P. Henríquez Ureña, "El español en Santo Domingo", $B D H, 5$ (1940), pp. 138 y 147 y "Mutaciones...", pp. 339-340.

7 Véase la nota 1 de Alonso y Rosenblat, al $\$ 154$ de A. Espinosa, "Estudios..." 
ese $51.88 \%$ de casos de $s$ final de palabra seguida de palabra que empieza con vocal. $\mathrm{Y}$ parece ser la misma razón por la cual, si bien no muy numerosa, se da la aspiración en posición explosiva (intervocálica o inicial absoluta).

4) Hay aspiración de $s$ intervocálica y es tan frecuente como la aspiración de $s$ final absoluta. La aspiración, o la relajación velar de $s$ intervocálica o inicial de palabra no es frecuente en el español de América y ni aun en el español de España. En América se da en Nuevo México (Espinosa, $\$ 154)^{8}$, en Chile ${ }^{9}$, raras veces en Colombia ${ }^{10}$ y parece ser que en Puerto Rico ${ }^{11}$, aunque Matluck lo niega rotundamente ${ }^{12}$; poco documentada se encuentra en Argentina y Bolivia ${ }^{13}$. En España se da en Andalucía y Sierra de Gata. En Canarias se realiza en algunos casos ${ }^{14}$. Matluck escribe que "se aspira la $s$ intervocálica sólo en los lugares en que se aspira la final, pero, con la excepción de nohotroh, tiene mucha menos extensión que la $s$ aspirada final. En México, según las noticias de que disponemos, la $s$ intervocálica no se aspira en ninguna parte, aun donde se aspira la $s$ final". (MATLuck, op. cit., \$ 127). Mis materiales parece que contradicen en parte lo apuntado arriba, pues en esta región casi no se aspira la $s$ final (absoluta) y sí un poco la explosiva; ya Menéndez Pidal había recogido la noticia de la aspiración intervocálica en México al citar que en Tabasco se dice lapihero ${ }^{15}$; al respecto creo en justicia que debo aclarar que cuando yo trabajé en el estado de Tabasco realizando encuestas para "Zonas Dialectales" en dos localidades (Villahermosa y Frontera) no escuché tal realización; sin embargo, en el estado de Sinaloa es muy notable.

En conclusión, la $s$ de La Cruz - de acuerdo con estos datos, escasos ciertamente-, es predorsoalveolar convexa apoyada en los incisivos inferiores, o plana, o dental convexa. Se aspira sobre todo en posición implosiva ante consonante sonora $(81 \%)$, medianamenre ante vocal $(52 \%)$, en menor grado ante sorda $(37 \%)$ y poco en posición final absoluta $(19 \%)$. Se registró aspiración en posición explosiva: intervocálica, inicial o tras $r$. Por lo que sospecho que el

8 Véase también Matluck, op. cit., $\$ 126$, nota y $\$ \S 111,153$ y 155 ; Hills, "El español de Nuevo México", p. 18 y P. Henríquez Ureña, "Mutaciones", p. 354 .

9 Véase R. Lenz, "El español en Chile”, pp. 92 y 251-252.

10 L. Flórez, op. cit., $\$ 89.1$.

11 Véase J. L. Dillard, "Sobre algunos fonemas puertorriqueños", NRFH, 16 (1962), pp. 422-424.

12 T. Matluck, "Fonemas finales", pp. 333-334.

13 Véase Matluck, op. cit., $\$ 120$, nota 243 ; también B. E. Vidal de BattiNi, El habla rural de San Luis, p. 41 y Tiscornia, $B D H, 3$ (1937), p. 46.

14 Véase D. Catalán, "El español en Canarias", PFE, 239-280.

15 R. Menéndez Pidal, "Sevilla frente a Madrid”, $H A M$, pp. 99-165. 
fenómeno se ve favorecido por el contacto sonoro con consonante o vocal. El proceso creo que es relativamente moderno y supongo que no tiene raíces en los fenómenos de población o en el sustrato, por ser el primero muy disparejo en cuanto a fechas y conglomerados, y el segundo por ser completamente nulo. Hay indicios de que aspiran más los hombres que las mujeres y, dentro de esta división, más los jóvenes que los viejos. Como dato complementario pueden revisarse los cuadros estadísticos de los cuestionarios, que confirman lo apuntado anteriormente y que está basado en los datos proporcionados por el análisis de las grabaciones magnetofónicas.

Juan López Chávez

Universidad Nacional Autónoma de México.

El Colegio de México. 
GRABACIONES

I

\begin{tabular}{|c|c|c|c|c|c|c|}
\hline Posición & Alófono & $I$ & $I I$ & III & $I V$ & Promedio \\
\hline & $s$ & 87.99 & & 61.70 & 84.61 & $61.23 \%$ \\
\hline & $\mathrm{h}$ & 3.99 & 58.49 & 4.25 & 15.38 & $19.38 \%$ \\
\hline$-5 /$ & ș & & 7.54 & 34.04 & & $8.80 \%$ \\
\hline & 5 & & 33.96 & & & $7.92 \%$ \\
\hline & $\mathrm{s}^{\mathrm{n}}$ & 7.99 & & & & $2.64 \%$ \\
\hline & $\mathrm{h}$ & 63.63 & 87.09 & 90.00 & 82.60 & $81.25 \%$ \\
\hline$-s+C S n$ & $\mathrm{~s}$ & 36.36 & & & 17.39 & $12.50 \%$ \\
\hline & 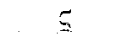 & & 9.67 & & & $3.12 \%$ \\
\hline & ș & & 3.22 & 10.00 & & $3.12 \%$ \\
\hline & $\mathbf{s}$ & 78.78 & & 50.82 & 46.15 & $40.98 \%$ \\
\hline & $\mathrm{h}$ & 21.21 & 58.00 & 22.95 & 48.71 & $37.70 \%$ \\
\hline$-s+C S r$ & ș & & 14.00 & 22.95 & 5.12 & $12.56 \%$ \\
\hline & 5 & & 28.00 & 1.64 & & $8.19 \%$ \\
\hline & $\dot{\mathrm{s}}$ & & & 1.64 & & $0.54 \%$ \\
\hline & h & 48.48 & 62.16 & 47.62 & 40.00 & $51.88 \%$ \\
\hline$-s+v$ & $\mathrm{~s}$ & 51.51 & & 38.09 & 60.00 & $32.07 \%$ \\
\hline & 5 & & 37.83 & 1498 & & $\begin{array}{r}13.20 \% \\
9830\end{array}$ \\
\hline & & & & & & \\
\hline & s & 97.29 & & 33.33 & 49.09 & $41.20 \%$ \\
\hline & 5 & & 72.00 & 10.52 & & $21.10 \%$ \\
\hline$-\mathrm{s}-$ & ș & & 6.00 & 43.85 & 16.36 & $18.59 \%$ \\
\hline & $\mathrm{h}$ & 2.70 & $22.00^{\circ}$ & 10.52 & 30.90 & $17.58 \%$ \\
\hline & $\mathrm{s}$ & & & & 3.63 & $1.00 \%$ \\
\hline & $\dot{\mathrm{s}}$ & & & 1.75 & & $0.50 \%$ \\
\hline & & & II & & & \\
\hline & $\mathrm{s}$ & 97.56 & 2.32 & 40.47 & 82.60 & $51.67 \%$ \\
\hline s- & 5 & & 72.09 & 38.09 & & $31.54 \%$ \\
\hline & $\mathrm{h}$ & 2.43 & 23.25 & 4.76 & 13.04 & $10.73 \%$ \\
\hline & ș & & 2.32 & 16.66 & 4.34 & $6.04 \%$ \\
\hline & s & 100 & & & 100 & $33.33 \%$ \\
\hline & ș & & 33.33 & 50.00 & & $25.00 \%$ \\
\hline rs & 5 & & 33.33 & & & $16.66 \%$ \\
\hline & $\mathrm{h}^{\mathrm{s}}$ & & 33.33 & & & $16.66 \%$ \\
\hline & $\dot{s}$ & & & 50.00 & & $8.33 \%$ \\
\hline & 5 & & 100 & 66.66 & & $58.82 \%$ \\
\hline ns & $\mathbf{s}$ & 100 & & 11.11 & 66.66 & $23.52 \%$ \\
\hline & $\dot{\mathrm{s}}$ & & & 22.22 & & $11.76 \%$ \\
\hline & $s^{\prime}$ & & & & 33.33 & $5.88 \%$ \\
\hline & 5 & & 75.00 & 100 & & $50.00 \%$ \\
\hline s5- & s & 100 & & & 100 & $37.50 \%$ \\
\hline & $\mathrm{h}$ & & 25.00 & & & $12.50 \%$ \\
\hline
\end{tabular}




\begin{tabular}{|c|c|c|c|c|c|}
\hline \multirow[b]{2}{*}{ Posición } & \multicolumn{4}{|c|}{ CUESTIONARIOS } & \multirow[b]{2}{*}{ Promedio } \\
\hline & Alófono & 1 & $I I$ & $I I I$ & \\
\hline \multirow{4}{*}{$-\$ /$} & $\mathbf{s}$ & 63.49 & 61.40 & 100.00 & $76.06 \%$ \\
\hline & $\mathrm{h}$ & 33.33 & & & $11.17 \%$ \\
\hline & ș & 3.17 & 19.29 & & $6.91 \%$ \\
\hline & s & & 19.29 & & $5.85 \%$ \\
\hline \multirow{3}{*}{$S+C S n$} & $\mathrm{~h}$ & 92.85 & 64.00 & 8.33 & $57.14 \%$ \\
\hline & $\mathrm{s}$ & 3.57 & 36.00 & 91.66 . & $41.55 \%$ \\
\hline & ș & 3.57 & & & $1.29 \%$ \\
\hline \multirow{4}{*}{$S+C S r$} & $\mathrm{~s}$ & 46.87 & 52.00 & 100.00 & $62.82 \%$ \\
\hline & $\mathrm{h}$ & 50.00 & 32.00 & & $30.76 \%$ \\
\hline & $\mathrm{s}$ & & 12.00 & & $3.84 \%$ \\
\hline & 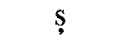 & 3.12 & 4.00 & & $2.56 \%$ \\
\hline \multirow{4}{*}{$-s-$} & $\mathbf{s}$ & 85.00 & 73.91 & 100.00 & $85.93 \%$ \\
\hline & $\mathbf{s}$ & & 17.39 & & $6.25 \%$ \\
\hline & ș & 10.00 & 8.69 & & $6.25 \%$ \\
\hline & $x^{s}$ & 5.00 & & & $1.56 \%$ \\
\hline \multirow{3}{*}{ s- } & $\mathbf{s}$ & 100.00 & 77.77 & 57.57 & $76.82 \%$ \\
\hline & $s^{\prime}$ & & 11.11 & 46.42 & $19.51 \%$ \\
\hline & s & & 11.11 & & $3.65 \%$ \\
\hline rs & $\mathbf{s}$ & 100 & 100 & 100 & $100 \%$ \\
\hline \multirow[t]{2}{*}{ ns } & s & 100 & 40.00 & 100 & $81.25 \%$ \\
\hline & s & & 60.00 & & $18.75 \%$ \\
\hline
\end{tabular}

